\title{
QUADRIFILAR HELIX ANTENNA FOR WEATHER SATELLITE RECEPTION
}

\author{
ENOCH ADEAGBO ${ }^{1,2 *}$, WALIU APENA ${ }^{1,3}$, KAYODE AKINGBADE $^{1,2}$ \\ ${ }^{1}$ Department of Electrical and Electronics Engineering, Federal University of Technology, \\ Akure, Nigeria \\ ${ }^{2}$ ICT Department, Elizade University, Ilara-Mokin, Ondo State, Nigeria \\ ${ }^{3}$ Department of Computer Engineering, Federal University of Technology, Akure, Nigeria
}

\begin{abstract}
The study designed and implemented quadrifilar helix antenna (QHA) for weather satellite signal reception. The antenna design and optimization were done by varying the element diameter, the radial lengths, the axial lengths, and element materials on adopted model. The simulated QHA has far-field radiation pattern in the upper hemisphere with maximum gain of $4.14 \mathrm{dBi}$ at $0^{\circ}$ and omnidirectional coverage, half-power beamwidth of $140^{\circ}$, bandwidth of $6.5 \mathrm{MHz}$, and VSWR of 1.13 . The implemented QHA has major lobe in conformity with the simulated QHA with maximum gain of $10.75 \mathrm{~dB}$ at $0^{\circ}$, and half-power beamwidth of $104^{\circ}$.
\end{abstract}

Keywords: Quadrifilar Helix Antenna (QHA), Numerical Electromagnetics Code (NEC2), Half-Power Beamwidth (HPBW), Low-Earth-Orbit satellites (LEOs), Circularly Polarized (CP), Voltage Standing Wave Ratio (VSWR)

\section{INTRODUCTION}

Polarization of an antenna is related to the orientations of electric fields radiated by the antenna. The Earth station equipped with a fixed receiving antenna would not be able to maintain polarization alignment with the low-Earthorbit satellites (LEOs) if the satellite antenna use linear polarization because of its ever-changing position. The circularly polarized (CP) antenna provides a solution to this problem and hence suitable for space communications [1]. CP antenna can reduce "Faraday rotation" effect due to ionosphere, the Faraday rotation effect causes about $3 \mathrm{~dB}$ or more signal loss if linearly polarized (LP) signals are employed [2]. The CP antenna is very effective in combating multi-path interference or fading [3,4]. Another advantage of $\mathrm{CP}$ antenna is that no strict orientation between transmitting and receiving antenna is required, also the strength of the received signals is fairly constant regardless of the antenna orientation with $\mathrm{CP}$.

Quadrifilar Helix Antenna (QHA) provides circular polarization and exhibits radiation pattern in the entire hemisphere. The radiation pattern is omnidirectional in the plane perpendicular to its main axis and thus provides fade-free reception. The basic form of the QHA was developed by Dr. C.C. Kilgus [5-7]. It usually consists of four half-turn helices (two bifilar helicals) equally spaced around the circumference of a common cylinder, the two bifilar helicals are arranged in a mutually orthogonal relationship along the common axis as in Figure 1. Kilgus

\footnotetext{
* Corresponding author, email: enoch.adeagbo@elizadeuniversity.edu.ng

(C) 2020 Alma Mater Publishing House
} 
showed that the resonant (element length $=\lambda / 2$ ), 1/2-turn, two bifilar helices, antiphased-fed, radiate a cardiodshaped, circularly polarized pattern when the diameter is $0.18 \lambda$ and the axial length is $0.27 \lambda$.

The variables and parameters used in the current and field analysis of the QHA are defined in Figure 1. The fields of the radials and the fields of the helical portions are evaluated independently and the summed. The assumed current distribution is sinusoidal with maxima at the feed and the distal end.

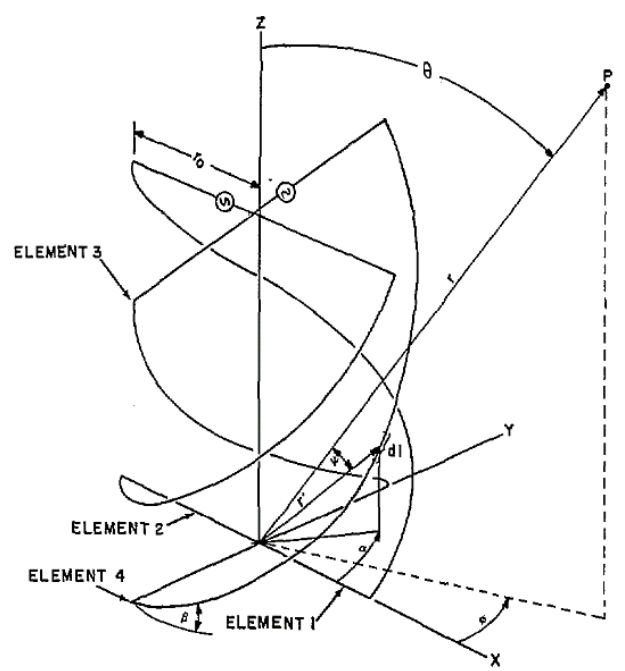

Fig. 1. Quadrifilar Helix Antenna [6].

The $\phi$ component of the total field of element 1 is:

$$
E_{\phi 1}=\frac{-j \omega \mu e^{-j k r}}{4 \pi r} \int_{l=0}^{l=\lambda / 2} i_{\phi} e^{j k r^{\prime} \cos \psi} d l
$$

For the field of the helical portions, let $\alpha$ be the integration variable:

$$
\begin{gathered}
d l=\frac{r_{0} d_{\alpha}}{\cos \beta} \\
E_{\phi H}=\frac{-j \omega \mu r_{0} e^{-j k r}}{4 \pi r \cos \beta} \int_{\alpha=0}^{2 N \pi} i_{\phi}(\phi, \alpha) e^{j k r^{\prime} \cos \psi} d \alpha
\end{gathered}
$$

For each element of the helix, the current magnitudes are:

$$
i_{\phi}(\phi)=I_{0} \cos \left(k r_{0}\right) \cos \frac{\alpha}{2 N} \cos \beta
$$

For elements 1 and 2:

$$
i_{\phi}(\phi, \alpha)=i_{\phi}(\alpha) \cos (\phi-\alpha)
$$

For elements 3 and 4 :

$$
i_{\phi}(\phi, \alpha)=i_{\phi}(\alpha) \sin (\phi-\alpha)
$$

The phase term for element 1 is:

$$
r^{\prime} \cos \psi=\frac{\overline{r^{\prime}} \cdot \bar{r}}{r}=r_{0} \cos \alpha \sin \theta \cos \phi+r_{0} \sin \alpha \sin \theta \sin \phi+\frac{P_{\alpha}}{2 \pi} \cdot \cos \theta
$$

Let: 


$$
K=\frac{\omega \mu I_{0} r_{0} \cos \left(k r_{0}\right) e^{-j k r}}{4 \pi r}
$$

Then $E_{\phi}$ for the helical portion of element 1 is given by:

$E_{\phi H 1}=-j K \int_{\alpha=0}^{2 N \pi} \cos \left(\frac{\alpha}{2 N}\right) \cdot \cos (\phi-\alpha) \exp \left[j k\left(r_{0} \cos \alpha \sin \theta \cos \phi+r_{0} \sin \alpha \sin \theta \sin \phi+\frac{P_{\alpha}}{2 \pi} \cdot \cos \theta\right)\right] d \alpha(5)$

Similarly, the field of element 2 is:

$$
E_{\phi H 2}=-j K \int_{\alpha=0}^{2 N \pi} \cos \left(\frac{\alpha}{2 N}\right) \cdot \cos (\phi-\alpha) \exp \left[j k\left(-r_{0} \cos \alpha \sin \theta \cos \phi-r_{0} \sin \alpha \sin \theta \sin \phi+\frac{P_{\alpha}}{2 \pi} \cdot \cos \theta\right)\right] d \alpha
$$

Elements 3 and 4 (the second bifilar helix) are fed in phase quadrature, to elements 1 and 2, respectively. The fields are:

$$
\begin{aligned}
& E_{\phi H 3}=K \int_{\alpha=0}^{2 N \pi} \cos \left(\frac{\alpha}{2 N}\right) \cdot \sin (\phi-\alpha) \exp \left[j k\left(-r_{0} \sin \alpha \sin \theta \cos \phi+r_{0} \cos \alpha \sin \theta \sin \phi+\frac{P_{\alpha}}{2 \pi} \cdot \cos \theta\right)\right] d \alpha(7) \\
& E_{\phi H 4}=K \int_{\alpha=0}^{2 N \pi} \cos \left(\frac{\alpha}{2 N}\right) \cdot \sin (\phi-\alpha) \exp \left[j k\left(r_{0} \sin \alpha \sin \theta \cos \phi-r_{0} \cos \alpha \sin \theta \sin \phi+\frac{P_{\alpha}}{2 \pi} \cdot \cos \theta\right)\right] d \alpha
\end{aligned}
$$

\section{Fields of the Radials}

If the current on the radials is approximated by a uniform distribution, the following simplified solutions result:

$$
\begin{aligned}
E_{\phi R 1,2} & =\frac{-j \omega \mu e^{-j k r}}{4 \pi r} 2 r_{0} I_{0} \cdot \sin \phi\left(e^{j k \cos \theta P / 2}-1\right) \\
E_{\phi R 3,4} & =\frac{\omega \mu e^{-j k r}}{4 \pi r} 2 r_{0} I_{0} \cdot \cos \phi\left(1-e^{j k \cos \theta P / 2}\right)
\end{aligned}
$$

\section{ANTENNA DESIGN AND IMPLEMENTATION}

There are design factors that determine how well an antenna system will function and thus impact the overall quality of the weather satellite imagery received. The design consists of controlling currents to produce the desired radiation pattern. The three design considerations of primary importance include:

- the physical size of the antenna components which is determined by the frequency of the transmissions it is intended to receive. In most Very High Frequency (VHF) antenna designs, the radiating elements are designed for $1 / 4$ or $1 / 2$ wavelengths:

- the antenna design should fit the type of RF signal polarization it is to receive;

- the antenna needs to provide enough signal gain to produce noise-free reception whenever it is used with an appropriate radio receiver.

The self-phased QHA design method which require only one coaxial cable for the feed, that is, a single excitation, was chosen over the hybrid-phased because the latter would require two feed lines to the bifilar loops, and would add loss and weight to the system. The self-phased is designed with different resonant lengths for the bifilar loops, fed in parallel resulting in 90-degrees current phase shift with respect to one another. This 90-degrees relationship is obtained by making their respective terminal impedances $R+j X$ and $R-j X$ where $X=R$, so that the currents in the respective helices are 45-degrees lag and 45-degrees lead. The relative lag and lead currents in the loops differ in phase by 90 -degrees does not require any additional components to obtain seperate differential phase excitations [8]. The resonant self-phased QHA, working group satellites was adopted as a model [9]. The diameter, axial length, and loop half-length of the larger bifilar are $0.148 \lambda, 0.330 \lambda$, and $0.560 \lambda$ respectively while the smaller bifilar are $0.141 \lambda, 0.314 \lambda$, and $0.528 \lambda$ respectively.

\subsection{The design of the QHA}

The design was carried out by varying the element diameter, the radial lengths, the axial lengths, and the element material for best performance. Numerical Electromagnetics Code (NEC2) was used for the antenna modeling. The radiating element wavelength is: 


$$
\begin{gathered}
\lambda=\frac{v}{f} \\
\lambda=\frac{3 \times 10^{8}}{137.5 \times 10^{6}}=2.1818 \mathrm{~m}
\end{gathered}
$$

were: $v$ is the speed of propagation of the wave in free space while $f$ is the operating frequency of the polar orbiting weather satellite of interest as given in Table 1.

Table 1. The Automatic Picture Transmission parameters for the polar operational environmental satellites of

$$
\text { U.S. [10]. }
$$

\begin{tabular}{|l|l|}
\hline Parameters & U.S. POES \\
\hline Frequency & $137.50,137.62,137.1,137.9125 \mathrm{MHz}$ \\
\hline Carrier Modulation & Analog AM/FM \\
\hline Transmit Power & 5 Watts \\
\hline Antenna Polarization & Right Hand Circular \\
\hline Carrier Deviation & $+/-17 \mathrm{KHz}$ \\
\hline
\end{tabular}

The dimension of the QHA is therefore. Larger bifilar:

$D=0.148 \lambda=322.90 \mathrm{~mm}$

$\ell_{p}=0.330 \lambda=719.99 \mathrm{~mm}$

$\ell_{E}=0.560 \lambda=1221.80 \mathrm{~mm}$

Smaller bifilar:

$D=0.141 \lambda=307.63 \mathrm{~mm}$

$\ell_{p}=0.314 \lambda=685.08 \mathrm{~mm}$

$\ell_{E}=0.528 \lambda=1151.99 \mathrm{~mm}$

where $D$ and $\ell_{p}$ are the diameter and axial length of the imaginary cylinder, and $\ell_{E}$ is the loop half-length.

The bifilar loops were segmented into 46 segments each to form straight-line wire segments and the wire segments $\mathrm{X}, \mathrm{Y}, \mathrm{Z}$ coordinates were inputted through the Geometry tab of the NEC editor following the QHA dimensions and the right-hand orientation as shown in Figure 2. Copper AWG0 (8.25246 mm diameter) wire element was selected as the wire-conductor and source excitation of 1volt was applied. The output data for the simulation shows that the QHA exhibits a far-field radiation pattern in the upper hemisphere and has omni-directional coverage sensitive in all directions with maximum gain of $4.37 \mathrm{dBi}$ at $0^{\circ}$ as in Figure 3 . The radiation pattern shows that the antenna would receive right-hand circular polarized waves from all directions (omnidirectional), thus the antenna can receive signal from the polar orbiting weather satellites. Figure 4 shows the main window with the antenna parameters.

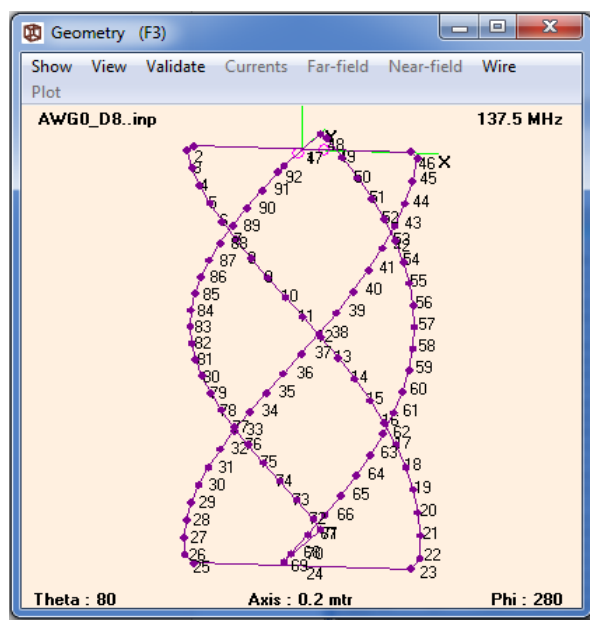

Fig. 2. The QHA Geometry.

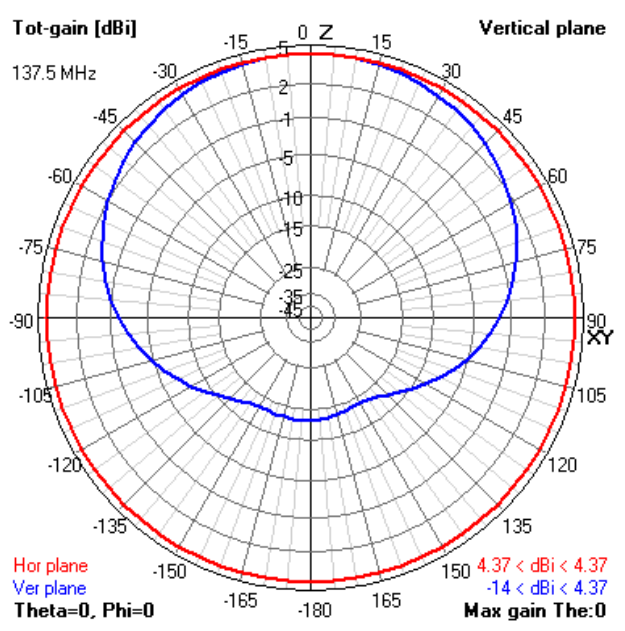

Fig. 3. Far-field radiation pattern of the QHA (Azimuth \& Elevation). 


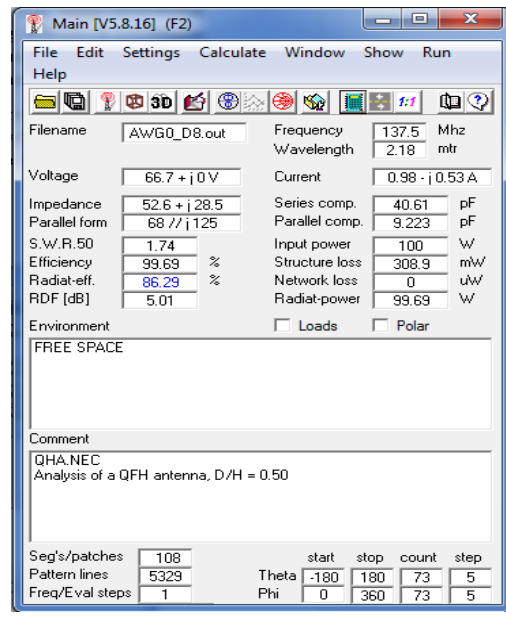

Fig. 4. The Main window with the antenna parameters.

From the main window, the antenna exhibits:

a. Impedance of $52.6+j 28.5$

b. VSWR of 1.74 .

c. Antenna Efficiency of $99.69 \%$.

d. Radiation-efficiency of $86.29 \%$.

e. Radiation-power of $99.69 \mathrm{~W}$.

\subsubsection{QHA Optimization}

NEC2 optimizer and evaluator was used to calculate the values of the element diameter, the radial lengths, and the axial lengths of the QHA that gave the best performance. The element diameter, and the radial lengths were varied at first and thereafter with the axial lengths. The element diameter, the radial lengths, and the axial lengths were replaced by variables with values extracted from the initial geometry inputs (Figure 5).

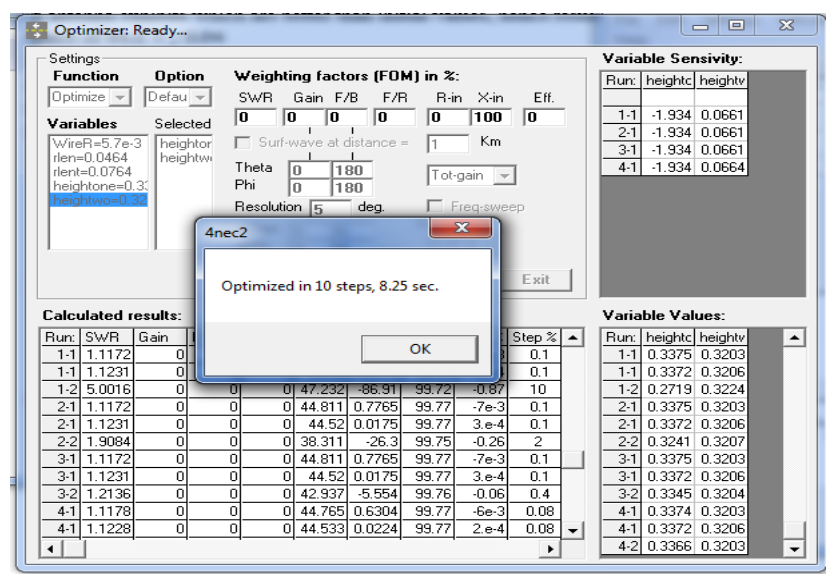

Fig. 5. The NEC2 optimizer and evaluator.

\subsection{The Implementation of the QHA}

Local materials were used for the antenna implementation such as: $3 / 8$ inches nominal size (12 $\mathrm{mm}$ diameter) copper tubing of about $7.5 \mathrm{~m}$ in length, 90-degrees $12 \mathrm{~mm}$ copper elbows (8 pieces), BNC RG58 $50 \Omega$ Cable, lead, and $32 \mathrm{~mm}$ PVC pipe with water proof end cap for the antenna support.

The optimized antenna dimension was used for the implementation following the geometry as in Figure 2. The length of the radials and the 1/2-turn elements were cut, the 1/2-turn loops were made by cutting out half-circle papers of radius that produced the circumferences of the loops and the element loops were curved along the circumference-path of the papers. The radials of the large and the small loops were arranged orthogonally, 90degrees to each other on the PVC pipe, the 1/2-turn elements were connected to the radials using the 90-degrees elbows following the right-hand circular polarization geometry. The use of a simple choke balun where the necessary isolating choke is formed from 4-turns winding of the feeder cable round the support mast was 
employed. Figure 6(a) shows the self-quadrature feeding method used [11] while Figure 6(b) shows the 4-turns winding choke balun [12]. Figure 7 shows the implemented right-hand circular polarized QHA.

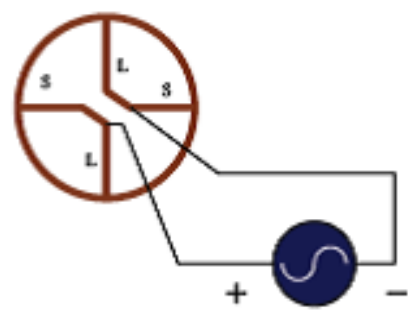

a.

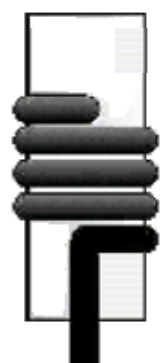

b.

Fig. 6. (a) Self-quadrature feeding method, L for long loop and S for small loop; (b) 4-turs winding choke balun.

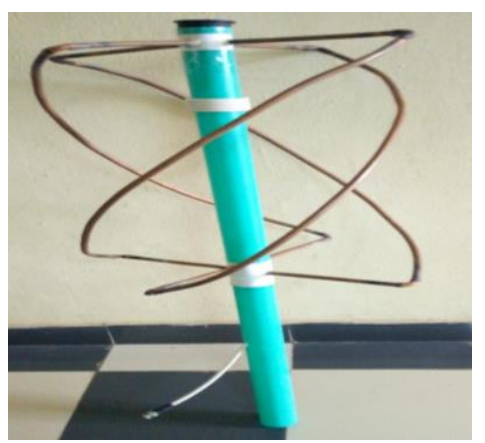

Fig. 7. The constructed right-hand circular polarized QHA.

\section{RESULTS AND DISCUSSION}

\subsection{The simulated QHA results}

The simulated results are provided in the following sections.

\subsubsection{QHA performance on element diameter}

The QHA performance on element diameter was simulated with different American Wire Gauge (AWG). The gains are:
a. AWG0000 (11.684mm diameter) delivered $-10<d B i<4.26$ gain;
b. AWG0 (8.25246mm diameter) delivered $-14<d B i<4.37$ gain;
c. AWG5 (4.62026mm diameter) delivered $-16<d B i<4.41$ gain;
d. AWG9 (2.90576mm diameter) delivered $-16<d B i<4.32$ gain;
e. AWG18 (1.02362mm diameter) delivered $-21<d B i<3.93$ gain.

The result shows that the diameter of the antenna element has effect on the radiation pattern and the gain(s) of the QHA as shown in Figure 8.

\subsubsection{QHA Performance on element diameter, radial lengths, and axial lengths}

Table 2 shows the final antenna optimization obtained from the QHA performance on element diameter, radial lengths, and the axial lengths.

Table 2. The results for the final antenna optimization.

\begin{tabular}{|l|c|c|}
\hline Variables & Initial variable value & New(optimized) variable \\
\hline Wire element radius (WireR) & 0.0041 & 0.005664 \\
\hline Bifilar element large (rlen) & 0.0788 & 0.046351 \\
\hline Bifilar element small (rlent) & 0.0746 & 0.076374 \\
\hline Bifilar axial length large (heightone) & 0.33715 & 0.336628 \\
\hline Bifilar axial length small (heightwo) & 0.3203 & 0.320317 \\
\hline
\end{tabular}




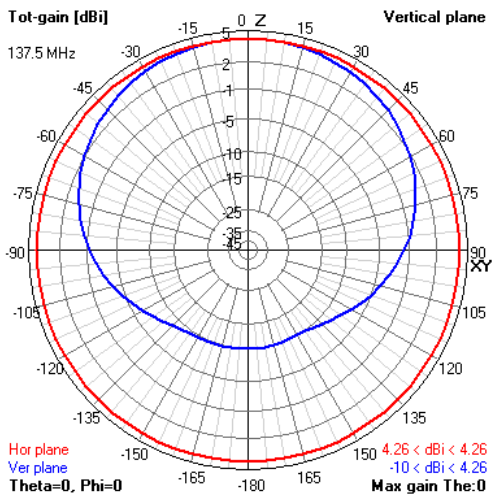

(a) AWG0000

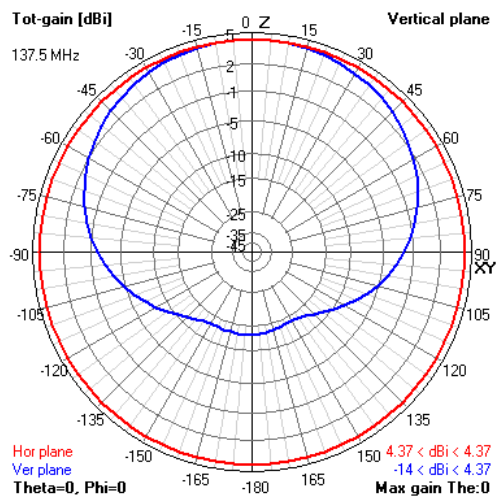

(b) AWG0

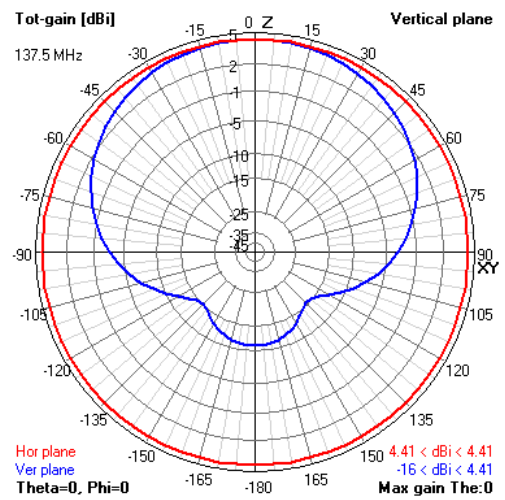

(c) AWG5

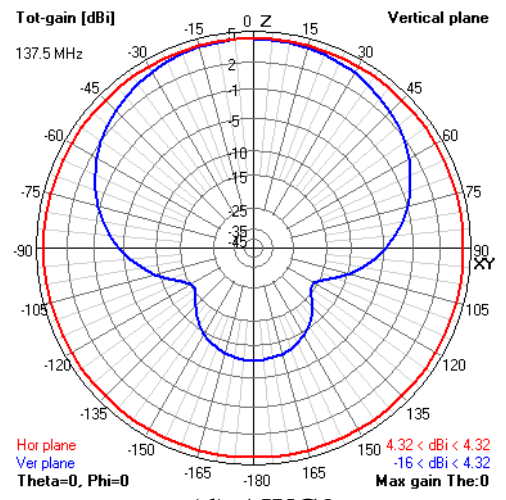

(d) AWG9

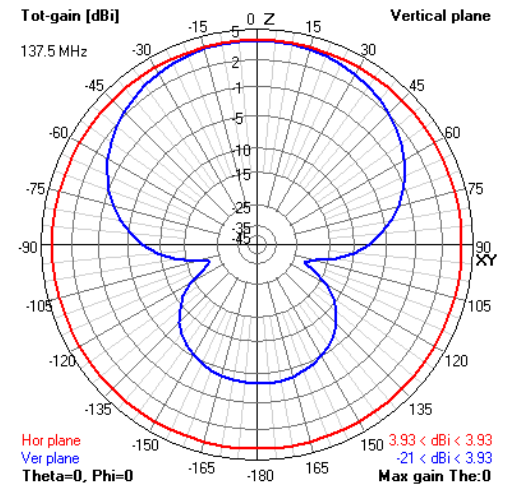

(e) AWG18

Fig. 8. Radiation pattern(s) and gain(s) of different copper wire guage.

\subsubsection{QHA performance on types of element materials}

Copper, Aluminum, Brass, Silver, and Phosphor-Bronze wire elements were tested to see their performances on the optimum dimension. The results obtained are presented in Table 3 and Figure 9, Figure 10, and Figure 11. The results showed that copper wire element has the best radiation efficiency, radiated power, minimal loss and best radiation pattern compared to Aluminum, Brass, Silver, and Phosphor-Bronze materials.

Table 3. The antenna gain (Reference $0 \mathrm{dBi}$ ) on different wire elements

\begin{tabular}{|l|l|l|l|l|l|l|l|}
\hline Elevation $(\theta)$ & $0^{\circ}$ & $45^{\circ}$ & $70^{\circ}$ & $90^{\circ}$ & $270^{\circ}$ & $315^{\circ}$ & $360^{\circ}$ \\
\hline Copper & 4.14 & 2.93 & 0.69 & -0.88 & -0.73 & 3.03 & 4.14 \\
\hline Aluminum & 4.14 & 2.93 & 0.69 & -0.89 & -0.74 & 3.03 & 4.14 \\
\hline Brass & 4.14 & 2.92 & 0.68 & -0.90 & -0.75 & 3.02 & 4.14 \\
\hline Silver & 4.14 & 2.93 & 0.69 & -0.88 & -0.73 & 3.03 & 4.14 \\
\hline Phosphor-Bronze & 4.13 & 2.92 & 0.67 & -0.91 & -0.76 & 3.02 & 4.13 \\
\hline
\end{tabular}

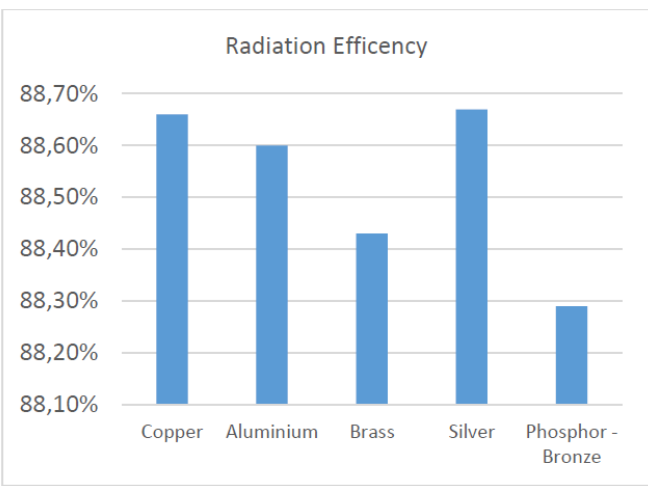

Fig 9. Radiation Efficiency. 


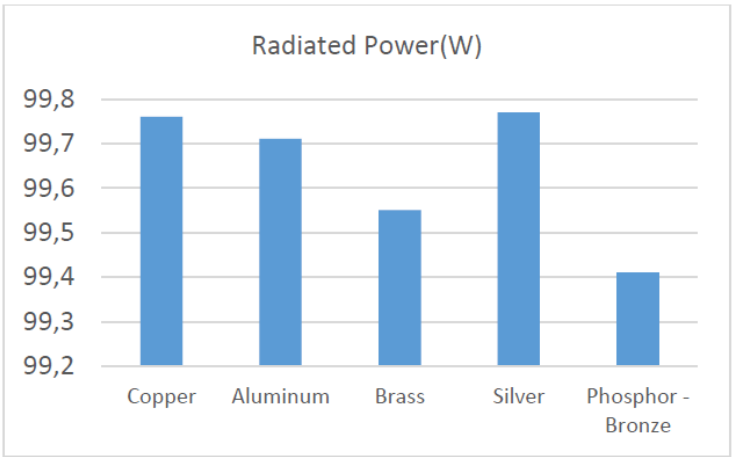

Fig 10. Radiated Power.

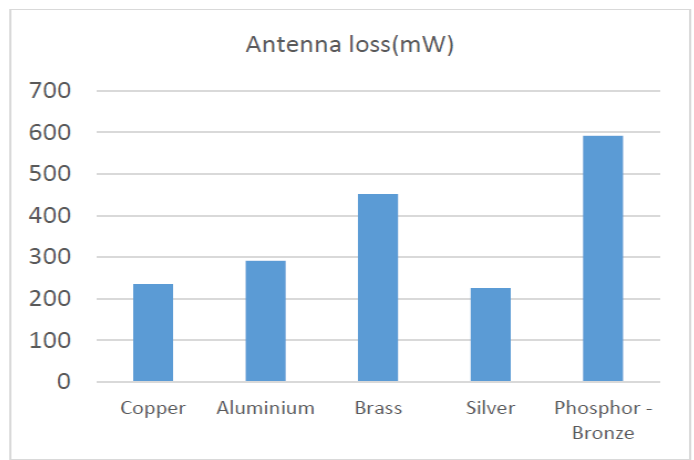

Fig 11. Antenna Loss.

\subsubsection{The optimized QHA dimensions and properties}

Using the "Wire/Segment info" on Geometry tab of NEC2, the geometry of the optimized QHA was calculated by summing up the wire segments as appropriate. Table 4 shows the optimized (new) dimensions and the nonoptimized (old) dimensions.

Table 4. The new (optimized) dimensions and the old (non-optimized) dimensions.

\begin{tabular}{|l|l|l|}
\hline & \multicolumn{1}{|c|}{ Optimized dimension } & \multicolumn{1}{c|}{ Non-optimized dimension } \\
\hline Larger loop & $D=338.40 \mathrm{~mm}=0.155 \lambda$ & $D=322.90 \mathrm{~mm}=0.148 \lambda$ \\
& $\ell_{p}=673.25 \mathrm{~mm}=0.308 \lambda$ & $\ell_{p}=719.99 \mathrm{~mm}=0.330 \lambda$ \\
& $\ell_{E}=1165.90 \mathrm{~mm}=0.534 \lambda$ & $\ell_{E}=1221.80 \mathrm{~mm}=0.560 \lambda$ \\
\hline Smaller loop & $D=305.50 \mathrm{~mm}=0.140 \lambda$ & $D=307.63 \mathrm{~mm}=0.141 \lambda$ \\
& $\ell_{p}=640.63 \mathrm{~mm}=0.293 \lambda$ & $\ell_{p}=685.08 \mathrm{~mm}=0.314 \lambda$ \\
& $\ell_{E}=1123.30 \mathrm{~mm}=0.514 \lambda$ & $\ell_{E}=1151.99 \mathrm{~mm}=0.528 \lambda$ \\
\hline
\end{tabular}

where $D$ and $\ell_{p}$ are the diameter and axial length of the imaginary cylinder, and $\ell_{E}$ is the loop half-length.

The optimized QHA has the following properties as shown in Figure 12 (a, b, c):

a. Far-field radiation pattern in the upper hemisphere (Elevation) with gains of $4.14 \mathrm{dBi}$ at $0^{\circ}, 2.93 \mathrm{dBi}$ at $45^{\circ}, 0.69 \mathrm{dBi}$ at $75^{\circ}$, and $-0.88 \mathrm{dBi}$ at $90^{\circ}$ and has omni-directional coverage sensitive in all directions (Azimuth) with gain of $4.14 \mathrm{dBi}$;

b. Half-power beamwidth of $140^{\circ}$. (It is the angle at which the antenna gain first falls to half power (approx. -3 $\mathrm{dB}$ ) from the peak. The angular distance between the half power points ( $-3 \mathrm{~dB}$ points $)$ is defined as the beamwidth); c. Impedance of $44.2+j 1.09$ (it was $52.6+j 28.5$ before optimization);

d. VSWR of 1.13 (it was 1.74 before optimization);

e. Antenna Efficiency of $99.77 \%$ (it was $99.69 \%$ before optimization);

f. Radiation-efficiency of $88.66 \%$ (it was $86.29 \%$ before optimization);

g. Radiation-power of $99.76 \mathrm{~W}$ (it was $99.69 \mathrm{~W}$ before optimization).

Frequency sweep was conducted on the antenna between $135 \mathrm{MHz}$ and $150 \mathrm{MHz}$, the following results were obtained: voltage standing wave ratio (VSWR) and reflection coefficient graph (Figure 13), gain graph (Figure 14), and the impedance graph (Figure 15). The designed frequency of $137.5 \mathrm{MHz}$ is at resonance where the impedance is purely (approximately) resistive.

\subsection{The Implemented QHA results}

Antenna Trainer ST2261 was used to measure the radiation pattern of the implemented antenna. The platform consists of the main unit, transmitting and receiving mast, RF detector and adapter, signal demodulator, accessories case containing antenna types, BNC to BNC 25 inches long cable for antenna connection and others. The main unit has RF generator, modulation generator, directional coupler, antenna rotation $\left(0-360^{\circ}\right)$ and others [13].

The setup for the measurement is shown in Figure 16 and below were the steps taking to plot the radiation pattern of the antenna. The implemented QHA was made the receiving antenna and put in a fix position pointed to the transmitting antenna:

- A right-hand helical antenna type was used as the transmitting antenna in order to provide the circular polarized wave which the QHA is designed for the transmitting antenna is attached to the antenna rotation provided; 
- Both transmitting and receiving antennas faced each other in horizontal plane and distance between them was about 1 meter as prescribed by the operating manual;

- The RF generator is turned on and the transmitter turned for maximum forward power for optimum radiation of the transmitting antenna;

- The FS adjust on the main unit is set at $10 \mu \mathrm{A}$ (and later $13 \mu \mathrm{A}$ for the second test) readings for the transmitting unit and the Digital Panel Meter (DPM) at RF detector unit attached to the receiving antenna is monitored for the corresponding output readings;

- The readings at intervals of 10 - degrees were taking. The ratio of $\mathrm{I}_{\mathrm{out}}$ and $\mathrm{I}_{\mathrm{in}}$, that is, the output readings at the receiving RF detector and the input reading of the transmitting DPM, are computed for the gain of the receiving antenna;

- To plot the radiation pattern of the receiving antenna, the gains at each degree are converted to decibels, $\{20 \log$ (gain) $\}$. The degrees versus the gains in $\mathrm{dB}$ are tabulated and used to plot the radiation pattern for the receiving antenna.

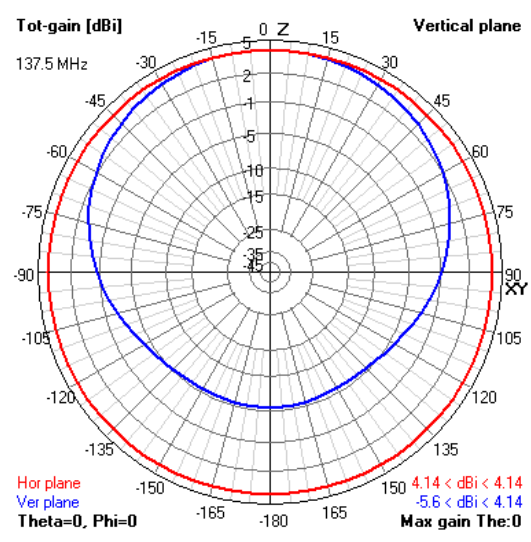

(a)

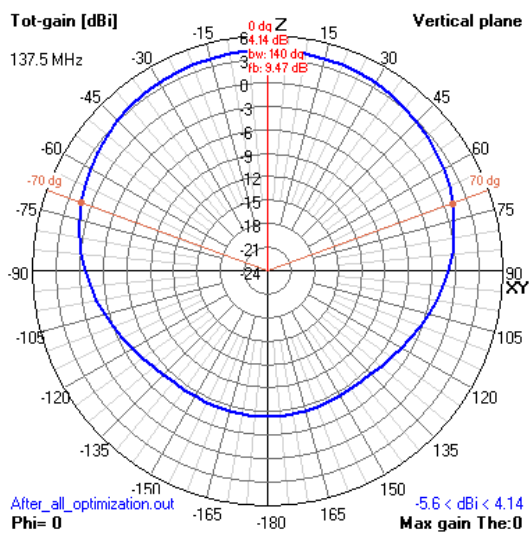

(b)

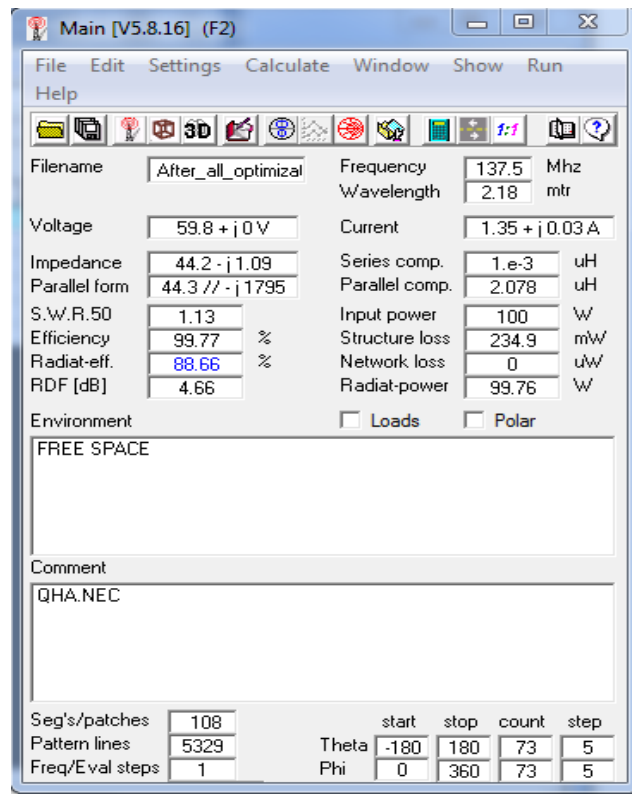

(c)

Fig. 12. (a) far-field radiation pattern of the optimized QHA (Azimuth and Elevation), (b) half-power beamwidth measurement, (c) the measured parameters. 


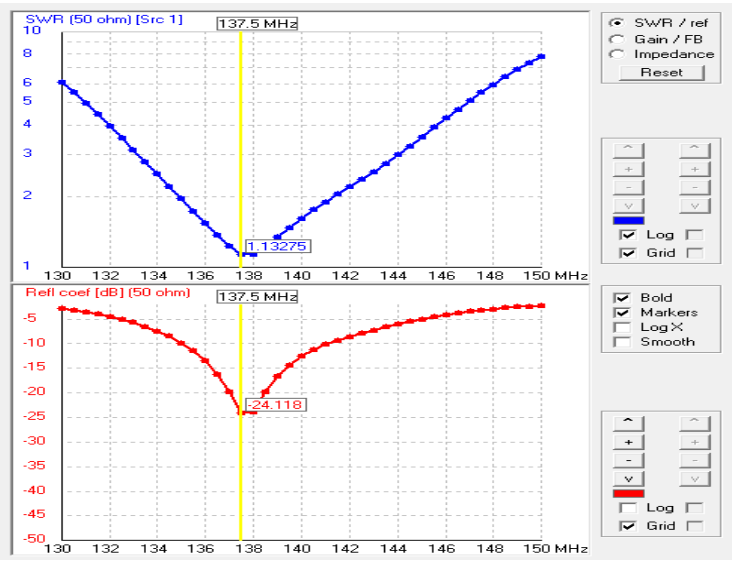

Fig. 13. The VSWR and the reflection coefficient graph.

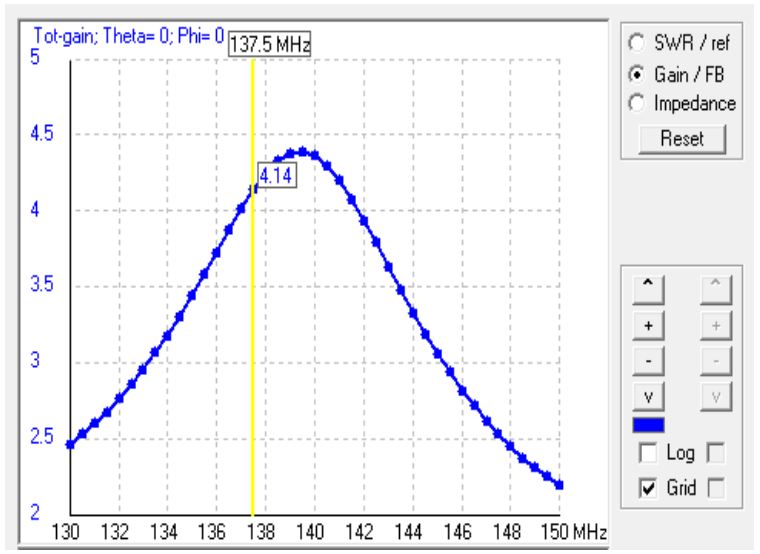

Fig. 14. The gain graph.

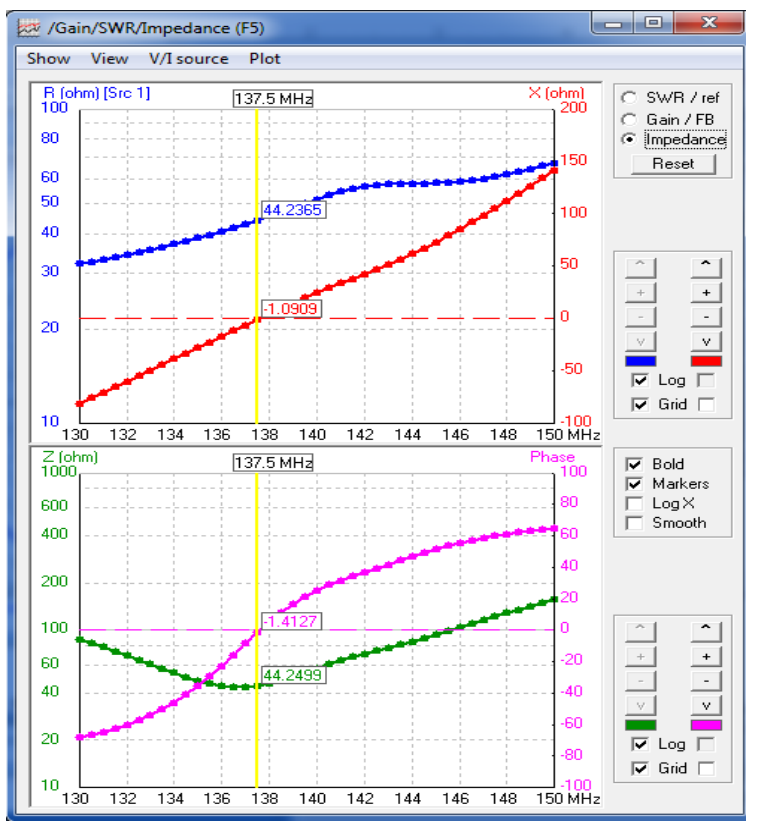

Fig. 15. The impedance graph.

The measurement obtained for the radiation pattern are plotted using Antenna Radiation Diagram Plotter 1.0.3 by R. Padovani, Figure 17.

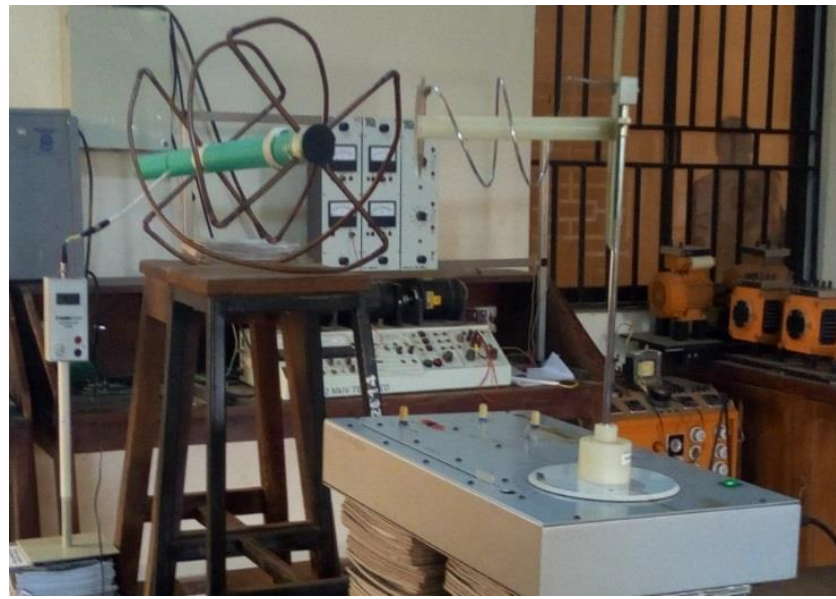

Fig. 16. QHA radiation pattern measurement setup using Antenna Trainer ST2261. 


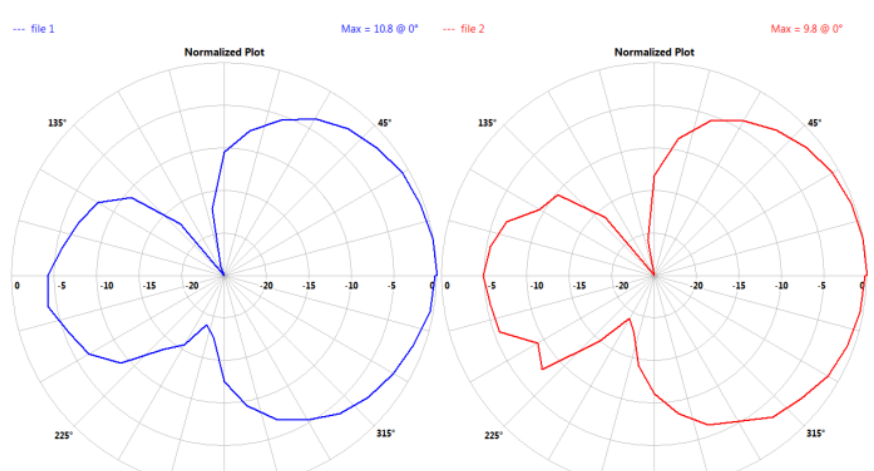

Fig. 17. The measured radiation pattern (Elevation) of the QHA constructed.

The implemented QHA has a major lobe as with the simulated QHA as expected. It has radiation pattern in the upper hemisphere (Elevation) with gains of $10.75 \mathrm{~dB}$ at $0^{\circ}, 8.26 \mathrm{~dB}$ at $50^{\circ}, 0.28 \mathrm{~dB}$ at $90^{\circ},-1.83 \mathrm{~dB}$ at $270^{\circ}, 7.88$ $\mathrm{dB}$ at $320^{\circ}$, and $10.51 \mathrm{~dB}$ at $360^{\circ}$, half-power beamwidth of $104^{\circ}$. The antenna also has back lobe which could be as a result of test environment electromagnetic wave influences, the antenna trainer kit limitations, and possible non-perfect geometry during implementation.

\subsection{Comparison between the simulated and the implemented QHA}

Comparison was done between the simulated and the implemented antenna. It was done with the parameters of the implemented antenna that could be measured within the available and availability of antenna measuring equipment, Table 5 shows the comparison done. It could be seen that the implemented antenna does not significantly deviate from the properties of the simulated version. The measured parameters are not expected to be same value but must follow same pattern most especially the radiation pattern and the gains.

Table 5. Comparison between the simulated and the implemented QHA.

\begin{tabular}{|l|c|c|}
\hline Parameters & Simulated QHA & Implemented QHA \\
\hline Gain(s) & $0^{\circ}-4.14 \mathrm{dBi}$ & $0^{\circ}-10.75 \mathrm{~dB}$ \\
& $30^{\circ}-3.60 \mathrm{dBi}$ & $30^{\circ}-9.90 \mathrm{~dB}$ \\
& $60^{\circ}-1.97 \mathrm{dBi}$ & $60^{\circ}-7.03 \mathrm{~dB}$ \\
& $90^{\circ}--0.88 \mathrm{dBi}$ & $90^{\circ}-0.28 \mathrm{~dB}$ \\
& $270^{\circ}--0.73 \mathrm{dBi}$ & $270^{\circ}--1.83 \mathrm{~dB}$ \\
& $300^{\circ}-2.09 \mathrm{dBi}$ & $300^{\circ}-5.24 \mathrm{~dB}$ \\
& $330^{\circ}-3.67 \mathrm{dBi}$ & $330^{\circ}-8.67 \mathrm{~dB}$ \\
& $360^{\circ}-4.14 \mathrm{dBi}$ & $360^{\circ}-10.51 \mathrm{~dB}$ \\
\hline HPBW & $140^{\circ}$ & $104^{\circ}$ \\
\hline Impedance & $44.2+j 1.09 \Omega$ & $50 \Omega$ (based on the impedance \\
& & of the feeding coaxial cable, the \\
& & balun, and the LNA impedance \\
& & to be used with the antenna \\
\hline
\end{tabular}

\section{CONCLUSIONS}

The study has provided platform in understanding the quadrifilar helix antenna design, its design optimization, and its implementation using local materials in receiving LEOs signals. The resonant QHA, Working Group Satellites was adopted as a model and the dimensions of the QHA was determined using the frequency of the transmissions the antenna is intended to receive. The design was carried out by varying the element diameter, the radial lengths, the axial lengths, and the element materials for antenna optimization. The simulation was carried out using NEC2, antenna modeling program.

The result shows optimized QHA dimension as \{diameter, $338.40 \mathrm{~mm}$; axial length, $673.25 \mathrm{~mm}$; loop halflength, $1165.90 \mathrm{~mm}$ \} for the larger bifilar and \{diameter, $305.50 \mathrm{~mm}$; axial length, $640.63 \mathrm{~mm}$; loop halflength, $1123.30 \mathrm{~mm}$ \} for the smaller bifilar, both with $11.328 \mathrm{~mm}$ wire element diameters at $137.5 \mathrm{MHz}$ (the resonant frequency of the transmissions the antenna is intended to receive). It could be seen from the results 
obtained that copper wire antenna element has the best radiation efficiency, radiated power, minimal antenna loss and best radiation pattern compared to Aluminum, Brass, Silver, and Phosphor-Bronze material.

The result shows optimized QHA dimension as \{diameter, $338.40 \mathrm{~mm}$; axial length, $673.25 \mathrm{~mm}$; loop halflength, $1165.90 \mathrm{~mm}$ \} for the larger bifilar and \{diameter, $305.50 \mathrm{~mm}$; axial length, $640.63 \mathrm{~mm}$; loop halflength, $1123.30 \mathrm{~mm}$ \} for the smaller bifilar, both with $11.328 \mathrm{~mm}$ wire element diameters at $137.5 \mathrm{MHz}$ (the resonant frequency of the transmissions the antenna is intended to receive). It could be seen from the results obtained that copper wire antenna element has the best radiation efficiency, radiated power, minimal structure loss and best radiation pattern compared to Aluminum, Brass, Silver, and Phosphor-Bronze material.

The implemented QHA was measured using antenna trainer ST 2261, it has major lobe as with the simulated QHA, that is, radiation pattern in the upper hemisphere (Elevation) with maximum gain of $10.75 \mathrm{~dB}$ at $0^{\circ}, 8.26 \mathrm{~dB}$ at $50^{\circ}, 0.28 \mathrm{~dB}$ at $90^{\circ},-1.83 \mathrm{~dB}$ at $270^{\circ}, 7.88 \mathrm{~dB}$ at $320^{\circ}$, and $10.51 \mathrm{~dB}$ at $360^{\circ}$. The antenna also has back lobe which could be as a result of test environment electromagnetic wave influences, the antenna trainer kit limitations, and possible non-perfect geometry during construction. It could be seen that the results available for the implemented QHA is approximately same as that of the simulated version.

\section{REFERENCES}

[1] Straw, R.D., Cebik, L.B., Hallidy, D., Jansson, D., Lewallen, R., Severns, R., Witt, F., The ARRL antenna book, $21^{\text {st }}$ edition, The ARRL Inc., Newington, USA, 2007, p. 4-1.

[2] Gao, S., Luo, Q., Zhu, F., Circularly polarized antennas, $1^{\text {st }}$ edition, John Wiley \& Sons Ltd, United Kingdom, 2014, p. 1-2.

[3] Balanis, C.A., Antenna Theory - Analysis and design, $2^{\text {nd }}$ edition, John Wiley \& Sons Inc., New York, 1997

[4] Maqsood, M., Dual band integrated antennas and arrays for global navigation satellite systems, PhD thesis. University of Surrey, 2012.

[5] Kilgus, C.C., Multi-element, fractional turn helices, IEEE Trans. AP-16, 1968, p. 499-500.

[6] Kilgus, C.C., Resonant quadrifilar helix, IEEE Trans. AP-17, 1969, p. 349-351.

[7] Kilgus, C.C., Spacecraft and ground station applications of the resonant quadrifilar helix, Digest of the 1974 Int. IEEE AP-S Symposion, 1974, p. 75-77.

[8] Bricker, R.W., Rickert, H.H., S-band resonant quadrifilar antenna for satellite communications, RCA Engineer vol. 20, no. 5, 1975, p. 78-82.

[9] Hollander, R.W., Resonant quadrifilar helix antenna, Working Group Satellites, 1999, p. 1-17.

[10] National Oceanic and Atmospheric Administration User's Guide for Building and Operating Environmental Satellite Receiving Stations U.S. Department of Commerce National Environmental Satellite, Data, and Information Service, Updated 2009.

[11] Slade, B., The basics of quadrifilar helix antennas, Technical Article, Orban Microwave, Orlando, 2015 , p. 14.

[12] Sykes, B., Cobey, B., Taming the quadrifilar helical antenna. www.askrlc.co.uk (9.07.2019).

[13] Scientech $2261 \quad$ (Antenna platform). http://link.scientechworld.com/ScientechLearning/Scientech2261/player.html (7.08.2019). 